\title{
MID-INFRARED EVIDENCE FOR ACCELERATED EVOLUTION IN COMPACT GROUP GALAXIES
}

\author{
Lisa May Walker ${ }^{1}$, Kelsey E. Johnson $^{1}$, Sarah C. Gallagher ${ }^{2}$, John E. Hibbard ${ }^{3}$, \\ Ann E. Hornschemeier ${ }^{4}$, Panayiotis Tzanavaris ${ }^{4}$ Jane C. Charlton $^{5}$, and Thomas H. Jarrett ${ }^{6}$ \\ ${ }^{1}$ Department of Astronomy, University of Virginia, Charlottesville, VA 22904, USA \\ 2 Department of Physics and Astronomy, University of Western Ontario, London, ON N6A 3K7, Canada \\ ${ }^{3}$ National Radio Astronomy Observatory, Charlottesville, VA 22903, USA \\ ${ }^{4}$ Laboratory for X-Ray Astrophysics, NASA Goddard Space Flight Center, Greenbelt, MD 20771, USA \\ ${ }^{5}$ Department of Astronomy and Astrophysics, Pennsylvania State University, University Park, PA 16802, USA \\ ${ }^{6}$ Spitzer Science Center, California Institute of Technology, Pasadena, CA 91125, USA \\ Received 2009 August 14; accepted 2010 August 24; published 2010 October 11
}

\begin{abstract}
Compact galaxy groups are at the extremes of the group environment, with high number densities and low velocity dispersions that likely affect member galaxy evolution. To explore the impact of this environment in detail, we examine the distribution in the mid-infrared (MIR) 3.6-8.0 $\mu \mathrm{m}$ color space of 42 galaxies from 12 Hickson compact groups (HCGs) in comparison with several control samples, including the LVL+SINGS galaxies, interacting galaxies, and galaxies from the Coma Cluster. We find that the HCG galaxies are strongly bimodal, with statistically significant evidence for a gap in their distribution. In contrast, none of the other samples show such a marked gap, and only galaxies in the Coma infall region have a distribution that is statistically consistent with the HCGs in this parameter space. To further investigate the cause of the HCG gap, we compare the galaxy morphologies of the HCG and LVL+SINGS galaxies, and also probe the specific star formation rate (SSFR) of the HCG galaxies. While galaxy morphology in HCG galaxies is strongly linked to position with MIR color space, the more fundamental property appears to be the SSFR, or star formation rate normalized by stellar mass. We conclude that the unusual MIR color distribution of HCG galaxies is a direct product of their environment, which is most similar to that of the Coma infall region. In both cases, galaxy densities are high, but gas has not been fully processed or stripped. We speculate that the compact group environment fosters accelerated evolution of galaxies from starforming and neutral gas-rich to quiescent and neutral gas-poor, leaving few members in the MIR gap at any time.
\end{abstract}

Key words: galaxies: clusters: general - galaxies: evolution - galaxies: interactions - galaxies: statistics - infrared: galaxies

Online-only material: color figures

\section{INTRODUCTION}

Galaxies form and evolve in a wide range of environments, from sparse field to densely populated groups and clusters. The most extreme densities are encountered in the cores of rich clusters, which are relatively rare in number, and in the more numerous compact groups (CGs; Hickson 1982). The Hickson compact groups (HCGs), owing to their high number density coupled with low velocity dispersions, undergo frequent tidal interactions, distortions, and mergers between group members (Hickson et al. 1992; Mendes de Oliveira \& Hickson 1994). HCGs are unique laboratories to study extreme galaxy evolution in the local universe and may also serve as analogs to galaxy formation and evolution in the early universe when population densities were much higher than what is observed in the field today.

A challenge of studying HCGs is moving from a morphologybased to a quantitatively based description of the star formation and evolution within the extreme population-density environment. Smith et al. (2007) find that the mass-normalized star formation rates (SFRs) in interacting galaxies are approximately twice that in normal spirals, lending support to the idea that interactions induce star formation. However, de la Rosa et al. (2007) and others find evidence of a mechanism that quenches the star formation in CGs. Specifically, they find that the stellar populations in elliptical galaxies in HCGs are more metal-poor and older than their counterparts in the field. Thus, although
CGs might be thought to be an ideal site for merger-induced star formation, this does not always seem to be the case.

In order to assess the impact of the CG environment on star formation, Johnson et al. (2007) looked at the Spitzer IRAC (3.6-8.0 $\mu \mathrm{m})$ color space distribution of HCGs and found that the mid-infrared (MIR) colors of galaxies in H I gas-rich HCGs are dominated by star formation, while the MIR colors of galaxies in $\mathrm{H}$ I gas-poor HCGs are dominated primarily by stellar photospheric emission, or are MIR-passive. From this, they infer an evolutionary sequence in which the gas in $\mathrm{H}$ I-rich groups is consumed, expelled, or ionized. In a complementary study of loose groups at higher redshift $(z \sim 0.4)$, Wilman et al. (2008) see a bimodality in $k$-corrected [ $f(8.0) / f(3.6)]$ colors, and a deficit of infrared activity when compared to field galaxies, similar to local H I-poor CGs. They note that the fraction of infrared excess galaxies, $f$ (IRE), decreases with galaxy stellar mass $M_{*}$, but within their group sample they see a deficit in $f$ (IRE) at all masses, and state that this trend can be explained if suppression of $M_{*}>10^{11} M_{\odot}$ galaxies occurs primarily in the group environment.

In Spitzer IRAC color-color plots of HCG galaxies, Johnson et al. (2007) noted a "gap" in the distribution of galaxies between those dominated by stellar light and galaxies that are actively star forming. This gap did not appear to be present in the initial comparison sample from the Spitzer First Look Survey (FLS). This comparison led them to speculate that the gap may be due to rapid evolution caused by the unique dynamical influences 
present in HCGs. However, the range in redshift of the FLS galaxies, which shifts polycyclic aromatic hydrocarbon (PAH) features in and out of the observing bands, renders the plot ambiguous. Nonetheless, Gallagher et al. (2008), using a related mid-IR diagnostic, confirmed the discrepancy by comparing HCG galaxies to the local SINGs sample (Dale et al. 2007), which like the FLS sample exhibits no gap in mid-IR color space. Tzanavaris et al. (2010) determined SFRs and specific SFRs (SSFRs) for this same HCG sample using Swift UV and Spitzer $24 \mu \mathrm{m}$ data and found a gap in SSFRs between $4.3 \times 10^{-12} \mathrm{yr}^{-1}$ and $1.6 \times 10^{-11} \mathrm{yr}^{-1}$. This gap did not exist in their comparison sample comprised of SINGS non-interacting and isolated galaxies. The fact that the HCG sample is discrepant with both the FLS and SINGS samples led them to conclude that the local environment in HCGs strongly influences the member galaxies.

Galaxies in CGs likely evolve differently than galaxies in other environments. Galaxies in clusters also experience frequent interactions, but the interaction timescale is shorter due to the large velocity dispersions, and they reside in a smoother gravitational potential, causing less torquing on the gas within galaxies. On the other hand, galaxies in loose groups experience less frequent interactions on average than those in CGs. Giant field galaxies experience fewer major interactions and frequently dominate their local gravitational potential. Comparing galaxies in high density environments (e.g., clusters, CGs) with galaxies in medium density environments (e.g., interacting pairs of galaxies) and low density environments (e.g., individual field galaxies) may allow greater understanding of how galaxy evolution is affected by the local density.

In an effort to learn more about the nature of the gap found by Johnson et al. (2007), we have examined the MIR colors of other samples of galaxies in a variety of environments. The previous comparison samples, FLS (Johnson et al. 2007) and SINGS (Gallagher et al. 2008; Tzanavaris et al. 2010), were both limited in scope and are known to suffer from issues with the samples that could have led to their discrepancy with the HCG sample. The goal of this paper is to expand the comparisons to a range of other samples with different properties in order to better assess the influence of environment on MIR color space distribution. We use the Kolmogorov-Smirnov (K-S) test to compare the MIR colors of the galaxies in these samples with those of the HCG galaxies to investigate to what extent the gap seen by Johnson et al. (2007) is ubiquitous, or unique to the CG environment.

\section{DATA/SAMPLES}

\subsection{HCG Galaxies}

The HCG data set, taken from Johnson et al. (2007), comprises 42 galaxies from 12 groups. The groups contain varying amounts of $\mathrm{H}$ I and span the three classification categories discussed in Johnson et al. (2007). The most H I gas-rich groups with $\log \left(M_{\mathrm{HI}}\right) / \log \left(M_{\text {dyn }}\right) \geqslant 0.9$ are classified as type I, the H I gas-poor groups with $\log \left(M_{\mathrm{HI}}\right) / \log \left(M_{\text {dyn }}\right)<0.8$ as type III, while type II contains the intermediate groups. It is also important to note that these categories reflect the group $\mathrm{HI}$, which is not necessarily simply the sum of the $\mathrm{HI}$ content of individual galaxies as this classification also includes gas in the intragroup medium. Table 1 gives background data on these groups. NED has been searched for known active galactic nuclei (AGNs), and four Seyfert IIs were identified: HCG 16b, 16d, 61a, and 90a. However, we expect emission from the AGNs in
Table 1

HCG Sample

\begin{tabular}{lccrrc}
\hline \hline HCG & $\begin{array}{c}\text { R.A. } \\
(\mathrm{J} 2000)\end{array}$ & $\begin{array}{c}\text { Decl. } \\
(\mathrm{J} 2000)\end{array}$ & $\begin{array}{c}D^{\mathrm{a}} \\
(\mathrm{Mpc})\end{array}$ & $\begin{array}{c}\log M_{\mathrm{H}_{\mathrm{I}}}^{\mathrm{b}} \\
\left(M_{\odot}\right)\end{array}$ & Group Type $^{\mathrm{c}}$ \\
\hline 02 & $0^{\mathrm{h}} 31^{\mathrm{m}} 30^{\mathrm{s}} .0$ & $+8^{\circ} 25^{\prime} 52^{\prime \prime}$ & 136.6 & 10.33 & I \\
07 & $0^{\mathrm{h}} 39^{\mathrm{m}} 23^{\mathrm{s}} .9$ & $+0^{\circ} 52^{\prime} 41^{\prime \prime}$ & 56.4 & 9.68 & II \\
16 & $02^{\mathrm{h}} 09^{\mathrm{m}} 31^{\mathrm{s}} .3$ & $-10^{\circ} 09^{\prime} 31^{\prime \prime}$ & 52.9 & $>10.42$ & I \\
19 & $02^{\mathrm{h}} 42^{\mathrm{m}} 45^{\mathrm{s}} .1$ & $-12^{\circ} 24^{\prime} 43^{\prime \prime}$ & 57.3 & 9.31 & II \\
22 & $03^{\mathrm{h}} 03^{\mathrm{m}} 31^{\mathrm{s}} .3$ & $-15^{\circ} 40^{\prime} 32^{\prime \prime}$ & 36.1 & 9.13 & II \\
31 & $05^{\mathrm{h}} 01^{\mathrm{m}} 38^{\mathrm{s}} .3$ & $-04^{\circ} 15^{\prime} 25^{\prime \prime}$ & 54.5 & 10.35 & I \\
42 & $10^{\mathrm{h}} 00^{\mathrm{m}} 21^{\mathrm{s}} .8$ & $-19^{\circ} 38^{\prime} 57^{\prime \prime}$ & 53.3 & 9.40 & III \\
48 & $10^{\mathrm{h}} 37^{\mathrm{m}} 45^{\mathrm{s}} .6$ & $-27^{\circ} 04^{\prime} 50^{\prime \prime}$ & 37.7 & 8.52 & III \\
59 & $11^{\mathrm{h}} 48^{\mathrm{m}} 26.6$ & $+12^{\circ} 42^{\prime} 40^{\prime \prime}$ & 54.1 & 9.49 & II \\
61 & $12^{\mathrm{h}} 12^{\mathrm{m}} 24.9$ & $+29^{\circ} 11^{\prime} 21^{\prime \prime}$ & 52.1 & 9.96 & I \\
62 & $12^{\mathrm{h}} 53^{\mathrm{m}} 08^{\mathrm{s}} .1$ & $-09^{\circ} 13^{\prime} 27^{\prime \prime}$ & 54.9 & $<9.06$ & III \\
90 & $22^{\mathrm{h}} 02^{\mathrm{m}} 06^{\mathrm{s}} .0$ & $-31^{\circ} 55^{\prime} 48^{\prime \prime}$ & 32.3 & 8.66 & III \\
\hline
\end{tabular}

Notes.

a Group distance taken from NED. The cosmology used was $H_{0}=$ $73 \mathrm{~km} \mathrm{~s}^{-1} \mathrm{Mpc}^{-1}, \Omega_{\text {matter }}=0.27$, and $\Omega_{\text {vacuum }}=0.73$.

${ }^{\mathrm{b}} \mathrm{H}$ I masses from Verdes-Montenegro et al. (2001), with the exception of HCG 90 , provided by J. Hibbard (2006, private communication).

${ }^{\mathrm{c}}$ Group type discussed in Section 2.1. Type I: H I-rich; Type III: H I-poor.

these cases should be minimal compared to the integrated light of the galaxy, as found by Gallagher et al. (2008).

One question brought up in discussions of CGs, especially HCGs, is the effect of selection biases. Mamon (1994) claims that the properties of CGs are a function of the algorithm used to find them. Ribeiro et al. (1998, p. 73) discuss Hickson's original selection criteria and how they influence the catalog of HCGs, such as ignoring the faint end of the luminosity function, selecting only groups with "significant surface density enhancement [...] over the field," and appearing to be well isolated, taking them out of context with their surroundings. Since Hickson's original catalog, several other catalogs of CGs have been published, some based on sky position and photometry like Hickson's, some utilizing redshift data. With the flood of data from SDSS, several catalogs of CGs have been generated, allowing selection effects to be qualitatively studied. Lee et al. (2004) selected CGs from the SDSS commissioning data using criteria slightly modified from Hickson's; they instituted an upper limit on number of group members, changed the isolation criteria to greater than three angular diameters, rather than greater than or equal to, and required higher surface brightness. They compared their catalog with six existing catalogs of CGs, including the HCGs, which they acknowledge as "the benchmark for all CG catalogs." For each CG catalog, they looked at the mean and median of group members, surface brightness, redshift, angular diameter, and linear diameter, and the HCGs tend to fall in the middle of each distribution (Table 3 in Lee et al. 2004). Thus, it appears that any selection biases that affect the HCGs are either more strongly present in other catalogs, or inherent properties of CGs as a class. In the latter case, this can be interpreted as reflecting the role of the CG environment on the evolution of the constituent galaxies.

\subsection{Comparison Samples}

Several comparison samples were selected from previous Spitzer IRAC studies. We have endeavored to include surveys that target a range of galaxy densities in order to begin to differentiate the role of environment on the observed MIR properties. These comparison samples include the LVL + SINGS galaxies (Dale et al. 2009, 2007), interacting galaxies (Smith 


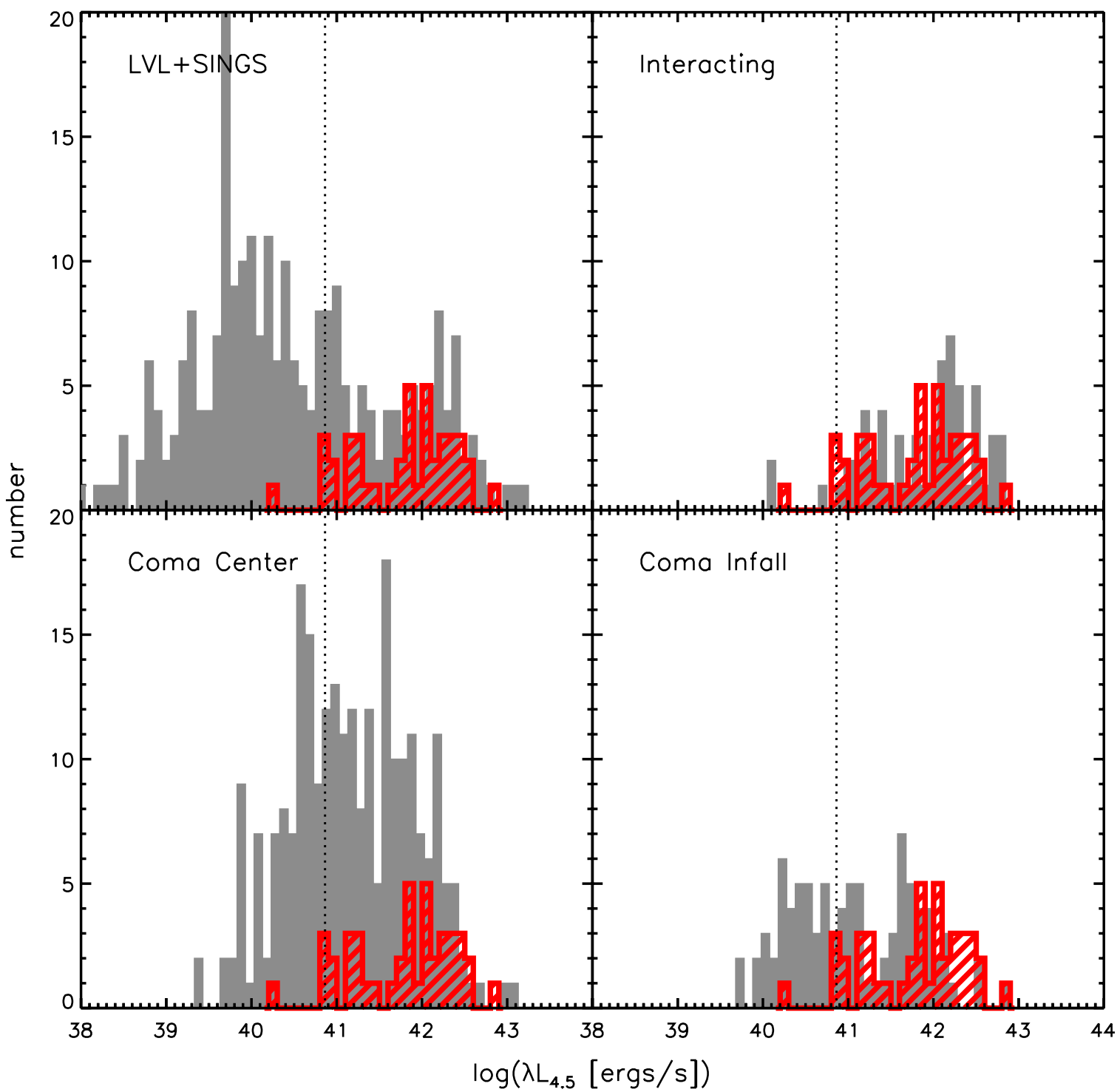

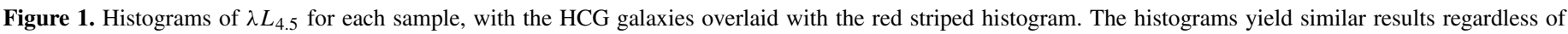
whether $\lambda L_{3.6}$ or $\lambda L_{4.5}$ is used. The dotted vertical line indicates the minimum luminosity for comparison and statistical analysis between samples.

(A color version of this figure is available in the online journal.)

et al. 2007), and galaxies in the Coma cluster (Jenkins et al. 2007). For both the HCG galaxies and the comparison samples, we only included galaxies at $z<0.035$, in order to ensure that the PAH features have not been redshifted into or out of their rest-frame IRAC channels.

The LVL data, discussed in Dale et al. (2009), consist of 258 galaxies within $11 \mathrm{Mpc}$. Galaxies with undefined flux values or only upper limits on the photometry were excluded, leaving 211 galaxies in the sample. The SINGS data, as presented and described in Dale et al. (2007), consist of 71 galaxies. Four of the galaxies in the original data set (M81 Dwarf A, NGC 3034, Holmberg IX, and DDO 154) were excluded because only upper or lower limits on the photometry were provided. It is important to note that the SINGS sample was chosen to be diverse, which will affect its distribution in color space. In addition, we combined the LVL and SINGS galaxies to create a control sample, referred to as LVL+SINGS. The 35 galaxies from the Spitzer Spirals, Bridges, and Tails Interacting Galaxy Survey (hereafter referred to as the interacting sample) are comprised of otherwise relatively isolated binary galaxy systems, whose members are tidally disturbed (Smith et al. 2007). This sample was biased toward galaxies with prominent signs of interaction, thereby selecting against elliptical galaxies. The Coma sample, discussed in Jenkins et al. (2007), is comprised of galaxies from two fields. The first field is located in the center of the cluster, where the galaxy density is very high. The second field is the infall region, located near 0.4 virial radii at the secondary X-ray peak, where the galaxy density is still higher than field density (Jenkins et al. 2007). Two galaxies from the center of the Coma cluster have been removed from the sample due to uncertain apertures, caused by proximity to one of the central elliptical galaxies. Our HCG sample has only one galaxy with luminosity below $\log \left(L_{4.5}\left[\mathrm{erg} \mathrm{s}^{-1}\right]\right)=40.9$. With Spitzer, we mapped the entire extent of each group to a $4.5 \mu \mathrm{m}$ sensitivity better than this limit (Johnson et al. 2007), thus our HCG database should be complete to this level. To be conservative, to compare the HCG galaxies to similar galaxy populations from the other samples we only consider galaxies with higher luminosities. The luminosity distributions of the samples are shown in Figure 1, with a vertical dotted line indicating the minimum luminosity 


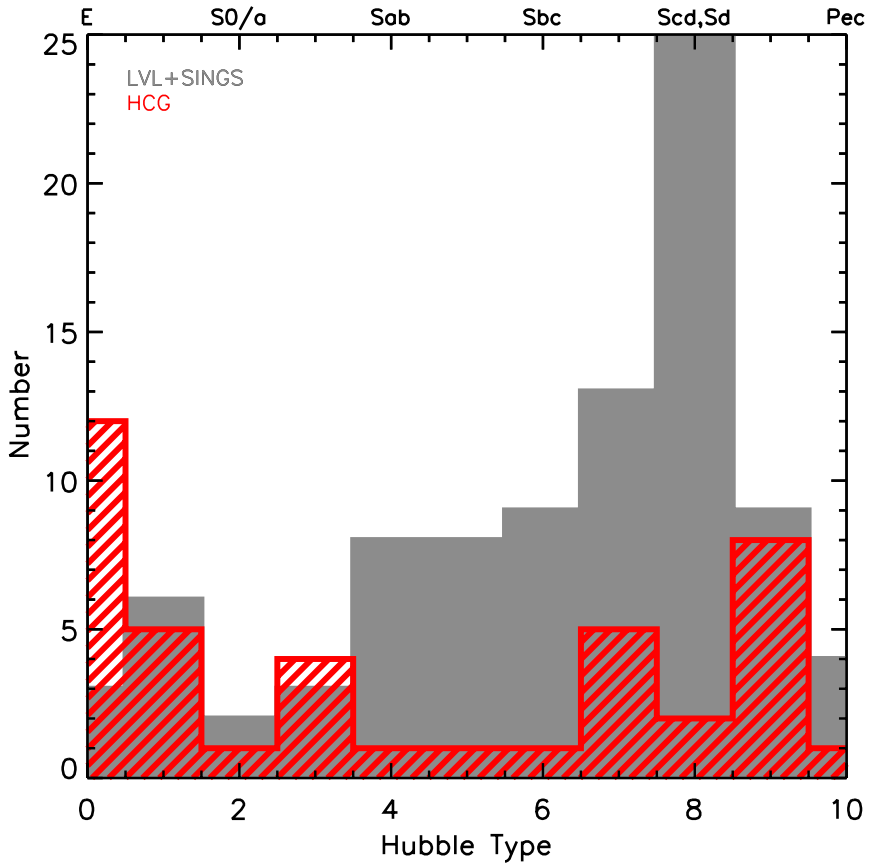

Figure 2. Histograms of Hubble type (following Haynes \& Giovanelli 1984) for the HCG galaxies (striped) and LVL+SINGS galaxies (solid).

(A color version of this figure is available in the online journal.)

required for inclusion. Figure 2 shows the distribution of Hubble types in our HCG sample compared with the LVL+SINGS sample. As can be seen in this plot, the LVL+SINGS sample is deficient in $\mathrm{Sa}$ galaxies, with respect to other Hubble types. The LVL+SINGS sample is dominated by spirals later than Sa, while the HCG sample contains galaxies across the distribution.

\section{MID-INFRARED COLOR SPACE}

\subsection{HCG Galaxies}

The IR color-color plot for the HCG galaxies is shown in Figure 3. The galaxies are separated into three types based on the fractional H I mass of the groups they belong to - the indigo plus signs represent type I ( $\mathrm{H}$ gas-rich), the red triangles indicate type II, and the blue squares correspond to type III (H I gas-poor). Galaxies in the lower-left portion of the plot have IR spectral energy distributions (SEDs) consistent with being dominated by stellar light. H I gas-poor groups fall in this region of the diagram due to the higher percentage of E/S0 galaxies. Galaxies with ongoing star formation will tend to fall in the upper right with red MIR colors indicative of interstellar PAH emission and thermal emission from warm/hot dust. The majority of the galaxies in the active region of the plot come from both type I (H I-rich) and type II groups, though there is a trend that galaxies from type II groups fall in the blue part of the active region, while the galaxies from type I groups show a larger range of colors throughout the region of the plot indicating activity. This trend can also be attributed to the percentage of E/S0 galaxies, as the type I groups contain very few E/S0 galaxies, while the type II groups have a varying percentage of $\mathrm{E} / \mathrm{S} 0$ galaxies.

As Johnson et al. (2007) noted, it is apparent in Figure 3 that the region between the galaxies dominated by stellar emission and galaxies with active star formation contains relatively few galaxies. In the following sections, we will explore the hypothesis that this gap in the MIR colors of HCG galaxies is due to rapid evolution through the stage during which

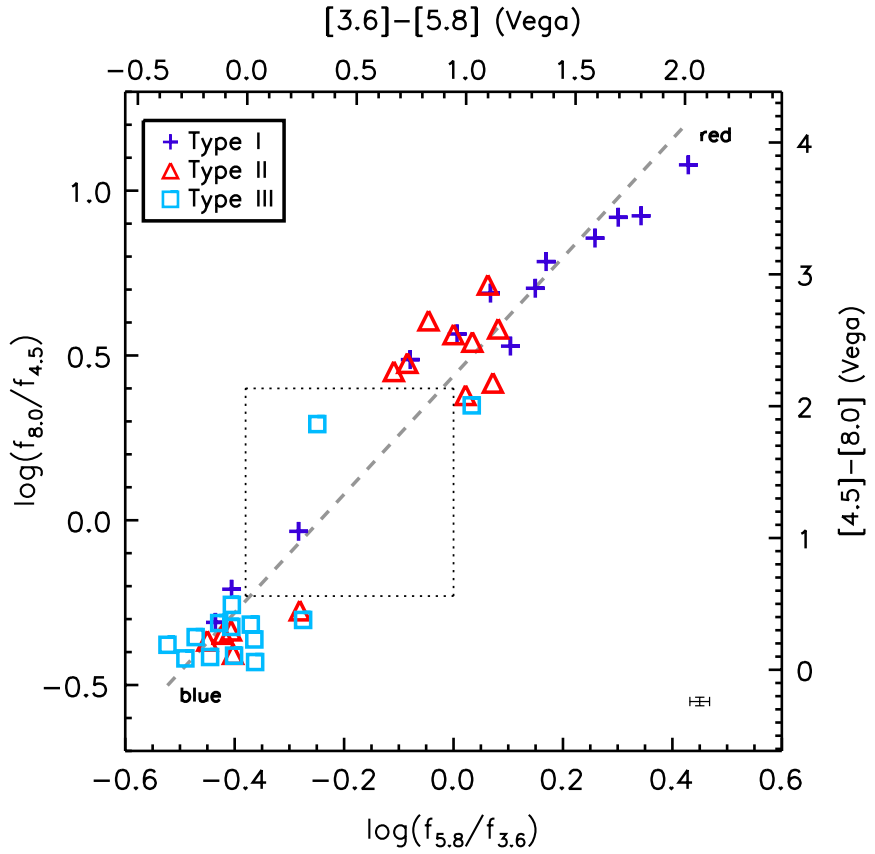

Figure 3. Color-color plot of HCG galaxies. The indigo plus signs are galaxies from type I (H I gas-rich) groups, the red triangles correspond to galaxies from type II groups, and the blue squares represent galaxies from type III (H I gaspoor) groups. The error bars in the lower right of the plot indicate typical errors for the sample. The lower left region of the plot contains galaxies whose light is dominated by stellar photospheric emission, while actively star-forming galaxies reside in the upper right. Between these is the "gap" noted by Johnson et al. (2007), indicated by the dotted box. The dashed line is the linear fit to the data, used for coordinate rotation in Section 4.

(A color version of this figure is available in the online journal.)

galaxies have intermediate MIR colors, or in other words, the HCG environment is biased against galaxies with very modest amounts of star formation.

\subsection{Comparison Samples}

The LVL+SINGS galaxies span the same region in MIR color space as the HCG galaxies, as shown on the top left in Figure 4. Given that the LVL is a volume-limited survey and SINGS galaxies were chosen to represent a range of physical properties and environments, it is not surprising that they are distributed relatively uniformly in color space rather than clustered in one region. Figure 4 does not show any evidence of a gap in color space for the LVL+SINGS galaxies. A plot made with the culled SINGS sample of Smith et al. (2007) (not shown) is similar, and also does not show a gap. The culled sample was created by removing galaxies with nearby companions from the SINGS sample.

The sample of interacting galaxies (top right plot of Figure 4) forms a very tight relationship in the star-forming region of color space, which is consistent with the fact that interactions frequently trigger star formation. There is only one point whose colors are consistent with being dominated by stellar populations, with little, if any, PAH emission and thermal emission from warm dust. The selection against elliptical galaxies mentioned in Section 2.2 also likely contributes to the lack of points in the blue region of IRAC color space.

The majority of the galaxies in the center of the Coma cluster lie in the region of MIR color space corresponding to stellar colors, with a smaller number of the galaxies scattered throughout the redward side of the plot. This is consistent with 


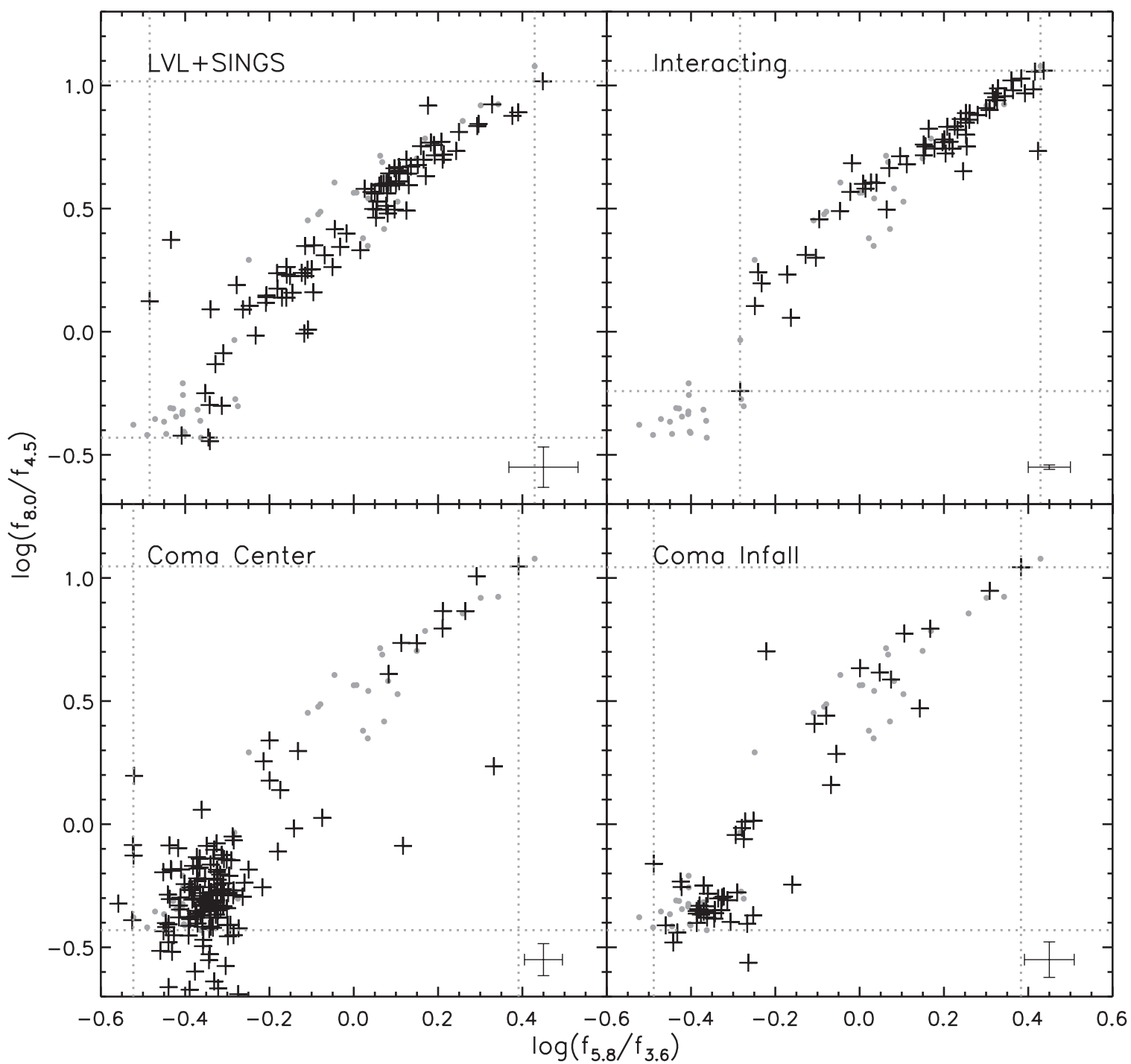

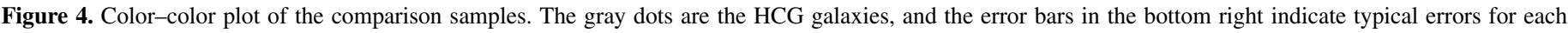

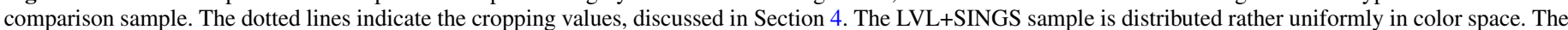

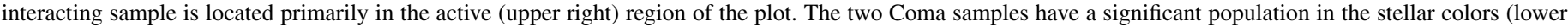

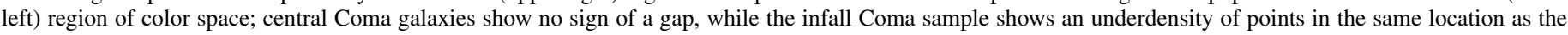
HCG gap.

the fact that galaxies in the cores of clusters tend to contain little to no $\mathrm{H}$ I and star formation. Unlike the other samples we consider, the central Coma galaxies do not follow a tight relationship in color space but rather have a much more scattered distribution. There are two galaxies with unusual colors: red $\log \left(f_{5.8} / f_{3.6}\right)$ and blue $\log \left(f_{8.0} / f_{4.5}\right)$. These two galaxies do not appear anomalous in the IRAC data, and we were unable to find anything in the literature which might explain their odd location in IRAC color space. However, both of these galaxies are in very crowded fields and that may be the cause of their odd colors. The central Coma galaxies are rather smoothly distributed, with no sign of a gap in their distribution.

Despite being from the same galaxy cluster, the distribution in color space of galaxies in the infall region of the Coma cluster is less concentrated in the region of stellar colors than the center of the Coma cluster. There are galaxies whose light is dominated by stellar emission, but the star-forming region for the infall sample is relatively more populated than the central Coma region. The color-color plot of galaxies in the infall region of the Coma cluster reveals an underdensity of points in the same location as the gap we see in the HCG galaxies. While it does not seem to be as pronounced, it may still carry some significance.

\section{STATISTICAL TESTS}

In order to assess whether the gap in the distribution of HCG galaxies seen in Figure 3 is significant, we perform a statistical analysis using the K-S test. The IDL routines ksone and kstwo were used to perform the K-S test, which first required rotating the data. The single-distribution version of the test allows comparison of each sample to a model of a uniform distribution and determines whether it is an accurate description of the data. The two-distribution version of the test compared two samples to determine whether they could have been drawn from the same distribution. Notably, it is only possible to conclusively reject the null hypothesis using this method; the K-S test cannot confirm the hypothesis.

We performed the two-distribution test in two ways: (1) comparing only the region in color space that is populated by both the HCG sample and the comparison sample (indicated by 
the dotted lines in Figure 4), from which we cannot draw any conclusions about the distributions outside of this range and (2) comparing the entire region of color space populated by either the HCG sample or the comparison sample. We fit a line to the HCG distribution in color space, which became the new $x$-axis in rotated color space, $\Delta C_{\mathrm{MIR}}$.

It is important to note that a "uniform" distribution does not necessarily mean a "normal" or "expected" distribution. A uniform distribution of galaxies in color space would be a sample whose galaxies fall evenly along a trend in color space. However, a normal distribution for old elliptical galaxies would show the galaxies preferentially in a clump in the bottom left, stellar colors region. In a normal distribution of active (starforming) galaxies, all the galaxies would fall linearly in the upper right, active region. What we wish to determine is which types of environments give rise to a gap.

\subsection{Single-distribution K-S Test}

For the single-distribution test, the cumulative distribution function $(\mathrm{CDF})$ of the rotated data was compared with the $\mathrm{CDF}$ of a model representing a uniform distribution along the $x$-axis over the same range as the galaxies. The resulting test yields two values: $D$, which is the maximum deviation of the data from the model, and $\alpha$, which gives the significance level with which it is possible to reject the null hypothesis that the model matches the data.

\subsubsection{HCG Galaxies}

Figure 5 shows the single-distribution K-S test for the HCG galaxies. It clearly illustrates the gap apparent in Figure 3, manifested as the nearly horizontal portion of the CDF over $-0.31<\Delta C_{\mathrm{MIR}}<0.38$ indicated by the gray rectangle. The maximum deviation of the sample from the model occurs at the beginning of the gap and is due to the pile-up of galaxies dominated by stellar light. The value of $\alpha$ returned by the test means that it is significant to reject the hypothesis that the HCG galaxies come from a uniform distribution at the $99.97 \%$ confidence level.

\subsubsection{Comparison Samples}

As Table 2 and Figure 6 show, the LVL+SINGS sample is mildly inconsistent with being drawn from a uniform distribution over the color range it covers. The CDF of the interacting sample is concave, indicating that the galaxies are dust-rich and likely gas-rich, forming stars. The CDF of center Coma galaxies, and to a lesser extent the galaxies in the infall region of Coma, is very convex. This is due to the relative overabundance of galaxies with stellar colors, caused by the lack of cold gas and therefore star formation present in these galaxies. Thus, the fact that the infall Coma region CDF is less convex than the center Coma region may indicate that galaxies in this region have not undergone as much processing. From the single-distribution test, it is clear that sample CDFs are affected by environment, as the HCG and Coma samples (i.e., the dense systems) have different CDFs from LVL+SINGS (i.e., the "field" sample).

\subsection{Two-distribution $K-S$ Test}

The two-distribution K-S test compared the CDF of each sample against the CDF of the HCG sample. This also calculated $D$ and $\alpha$, with the same meaning as the single-distribution test except that $D$ is now the maximum deviation between the two samples.

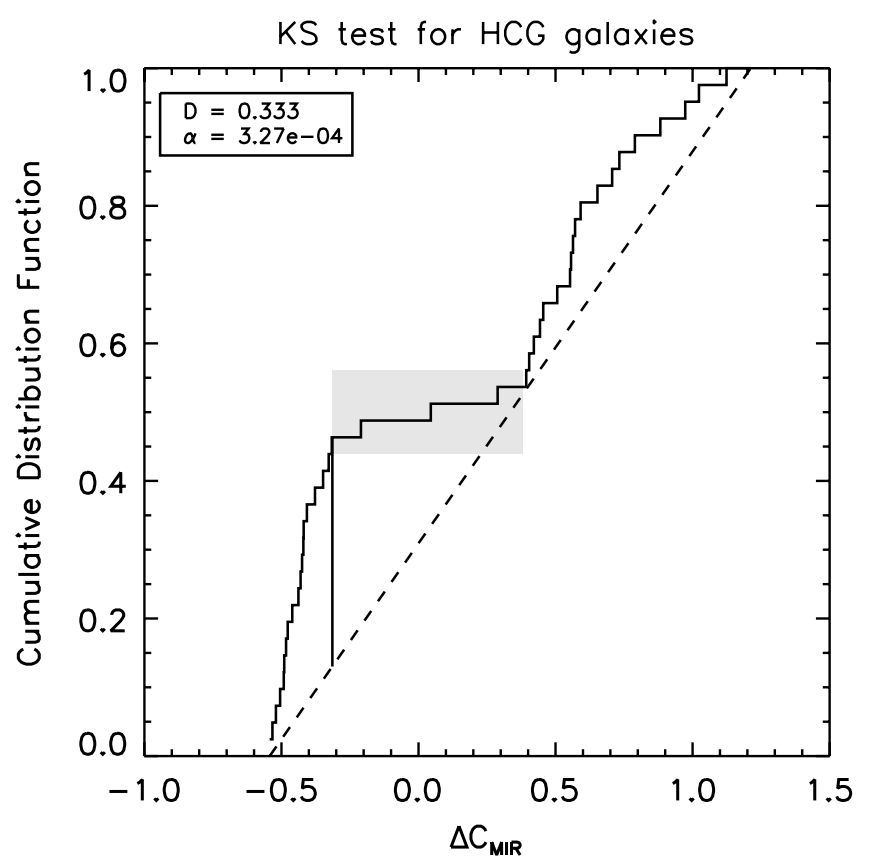

Figure 5. K-S test for the rotated HCG distribution against a model of uniform distribution. The maximum vertical distance between the CDF of the HCG galaxies (solid line) and that expected for a uniform distribution (dashed line) is $D$, indicated by the vertical line. The large value of $D$ indicates that the probability that the HCGs are drawn from a uniform distribution is very low, $0.03 \%$. The nearly flat portion of the CDF highlighted in gray qualitatively reveals the gap.

The results of the two-distribution K-S test are given in Table 3 and plotted in Figure 7. We deemed a sample inconsistent with the HCG sample if its probability of being consistent with the HCGs was less than $1 \%$. It is apparent that the distribution of galaxies in the center of Coma is drastically different from the distribution of HCGs, and the distributions for the LVL+SINGS galaxies and interacting galaxies are mildly inconsistent with being drawn from the same distribution as the HCGs. The $\alpha$ value for the infall Coma region sample is high enough that we cannot reject the hypothesis that it is drawn from the same parent distribution. Thus, the infall Coma region is most like the HCGs, as it has the smallest $D$ and largest $\alpha$.

\subsection{Gap Region}

In order to discover the depth of the gap seen in HCG color space, we devised a test to quantitatively discern whether any of the comparison samples exhibit a similar gap. We show the gap region by the gray box in Figure 5. This region was defined by the two HCG galaxies bounding the gap, at $\Delta C_{\mathrm{MIR}}=-0.31$ and 0.38 . Since the gap represents a deficit of galaxies over this color range, its signature is a flattening in the CDFs. Therefore, as another quantitative measure of the gap we performed a linear fit to the CDF between the color boundaries to obtain the slope; shallower slopes indicate samples with more pronounced gaps. The results are given in Table 4, which shows that the HCG sample has the most pronounced gap of any of the samples. We have excluded the interacting sample as it begins mid-gap (see Figure 4), so a slope would be undefined. The remaining three samples all contain galaxies in the region of stellar colors and thus cover the entire gap region. The slope of the CDF of the infall Coma region over the gap is fairly shallow, possibly indicating the existence of a less-defined gap than the HCG galaxies. 
Single-Distribution KS Test

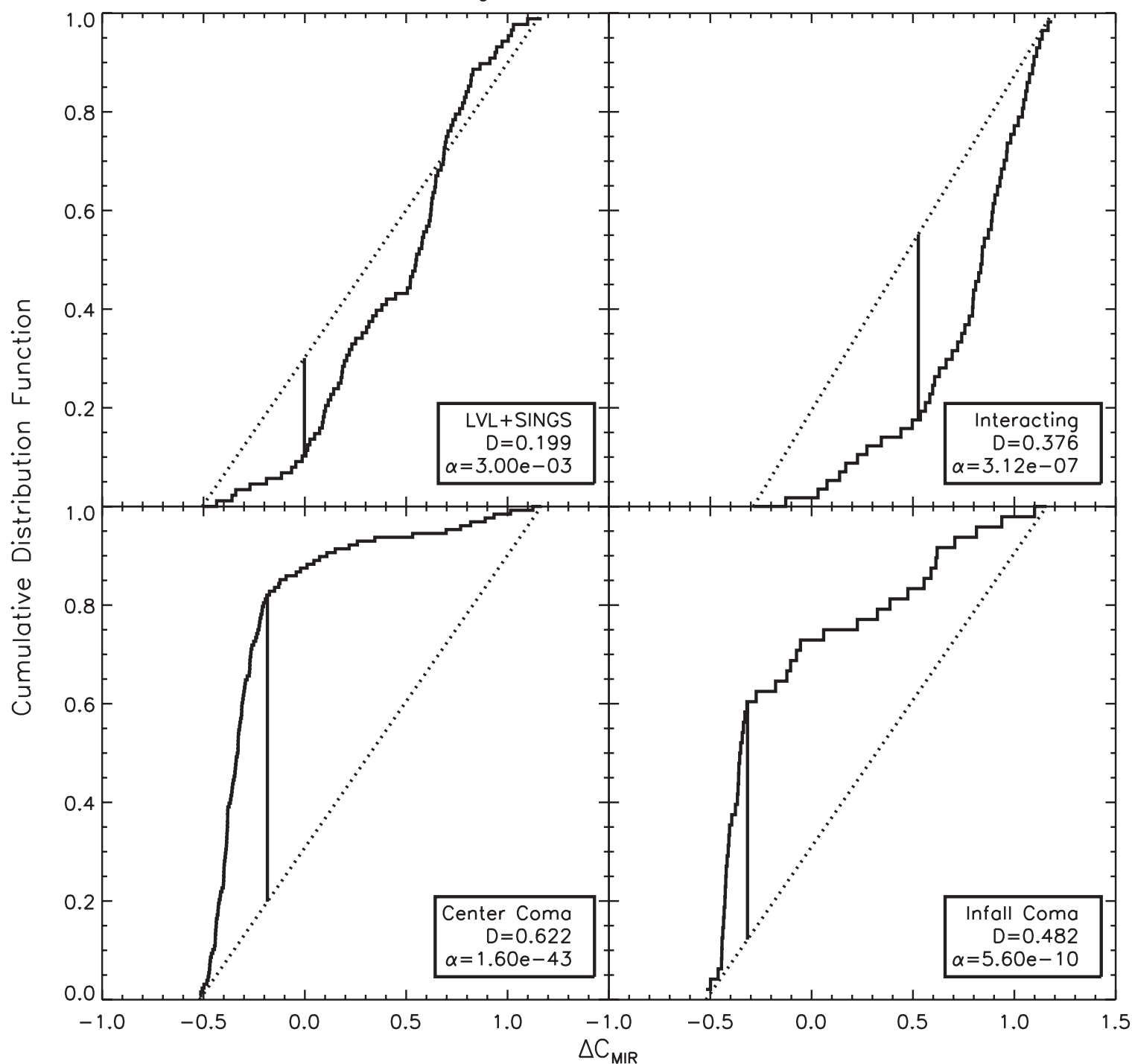

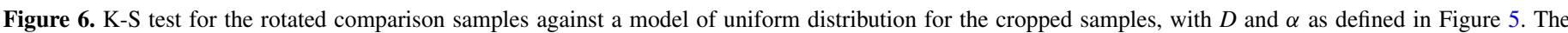

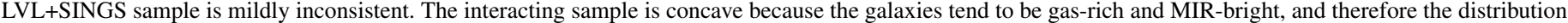
is weighted toward higher values of $\Delta C_{\mathrm{MIR}}$, while the two Coma samples are very convex due to the large fraction of galaxies with stellar colors in the samples.

Table 2

Single-distribution K-S Test

\begin{tabular}{|c|c|c|c|c|c|c|c|}
\hline \multirow[b]{2}{*}{ Sample } & \multicolumn{3}{|c|}{ Cropped } & \multicolumn{3}{|c|}{ Uncropped } & \multirow[b]{2}{*}{ Consistent w/Uniform } \\
\hline & $N$ & $D$ & $\alpha$ & $N$ & $D$ & $\alpha$ & \\
\hline $\mathrm{HCG}$ & $\ldots$ & $\ldots$ & $\ldots$ & 41 & 0.333 & $3.27 \times 10^{-4}$ & Reject \\
\hline LVL+SINGS (culled) & 67 & 0.236 & $1.72 \times 10^{-3}$ & 68 & 0.238 & $1.37 \times 10^{-3}$ & Do not reject \\
\hline Interacting & 57 & 0.376 & $3.12 \times 10^{-7}$ & 58 & 0.375 & $2.79 \times 10^{-7}$ & Reject \\
\hline Coma (center) & 128 & 0.622 & $1.60 \times 10^{-43}$ & 155 & 0.378 & $1.96 \times 10^{-19}$ & Reject \\
\hline
\end{tabular}

Note. The model uniform distribution covers only the range where galaxies are located and is different for each sample.

\section{COLOR-MAGNITUDE DIAGRAMS}

Optical color-magnitude diagrams have proven useful for understanding how galaxy luminosity (as a proxy for stellar mass) and color (as an indicator of the level of current star formation) are related. Galaxies are not evenly distributed in this parameter space, but are found in distinct regions: the so-called red sequence, blue cloud, and green valley (Hogg et al. 2004), discussed in Section 7.3. We are interested in investigating whether a color-magnitude diagram using only mid-IR information is similarly helpful in elucidating galaxy properties.

We created a plot analogous to a color-magnitude diagram using the data discussed in Section 4. A comparison to the 
Two-Distribution KS Test

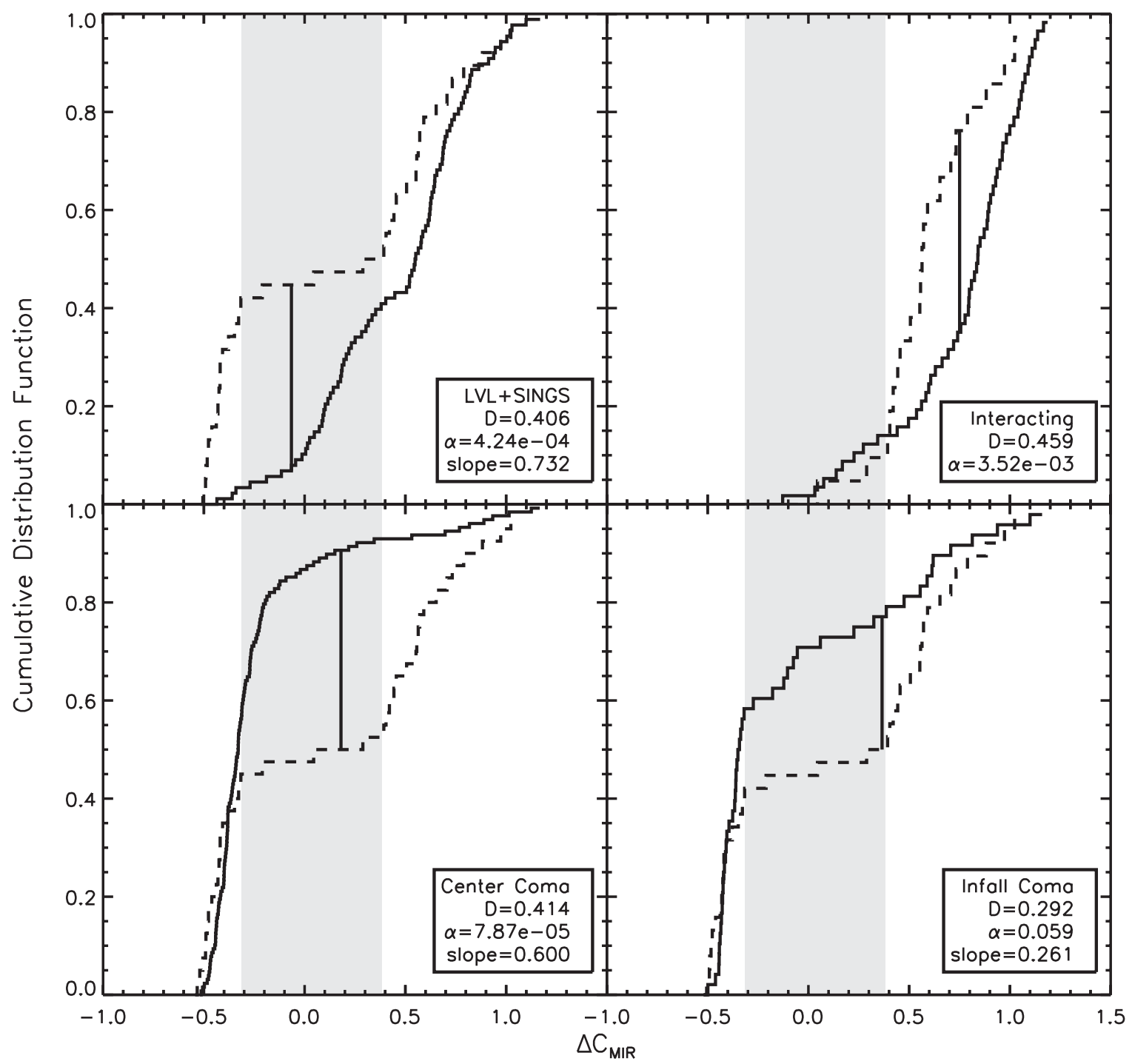

Figure 7. K-S test for the rotated comparison samples against the HCG galaxies for the cropped samples. From the large values of $D$ and small values of $\alpha$, as defined in Figure 5, the LVL+SINGS, interacting, and Coma center samples are clearly inconsistent with the HCG sample. The Coma infall sample has a high enough $\alpha$ that we cannot reject the hypothesis that it is consistent with the HCGs. The slope is a linear fit to the CDF over the gap region.

Table 3

Two-distribution K-S Test

\begin{tabular}{|c|c|c|c|c|c|c|c|c|}
\hline \multirow[b]{2}{*}{ Sample } & \multicolumn{3}{|c|}{ Cropped } & \multicolumn{3}{|c|}{ Uncropped } & \multirow[b]{2}{*}{ Consistent w/HCGs } & \multirow[b]{2}{*}{ Reason } \\
\hline & $\mathrm{N}_{\mathrm{HCGs}}$ & $D$ & $\alpha$ & $\mathrm{N}_{\text {HCGs }}$ & $D$ & $\alpha$ & & \\
\hline LVL+SINGS & 38 & 0.406 & $4.24 \times 10^{-4}$ & 41 & 0.399 & $3.42 \times 10^{-4}$ & Likely reject & $\overline{a, b}$ \\
\hline LVL+SINGS (culled) & 36 & 0.413 & $8.74 \times 10^{-4}$ & 41 & 0.429 & $2.11 \times 10^{-4}$ & Likely reject & $a, b$ \\
\hline Interacting & 21 & 0.459 & $3.52 \times 10^{-3}$ & 41 & 0.574 & $3.11 \times 10^{-7}$ & Reject & $\mathrm{b}, \mathrm{c}$ \\
\hline Coma (center) & 40 & 0.414 & $7.87 \times 10^{-5}$ & 41 & 0.441 & $9.01 \times 10^{-6}$ & Reject & $\mathrm{a}, \mathrm{c}$ \\
\hline Coma (infall) & 38 & 0.292 & 0.059 & 41 & 0.292 & 0.047 & Do not reject & $\mathrm{c}$ \\
\hline
\end{tabular}

Notes. Cropped covers only the region of color space that galaxies from both samples occupy. Uncropped covers the entire region of color space that galaxies from either sample occupy. Qualitative reasons for inconsistency: (a) no gap; (b) fundamentally different; (c) covers different range in color space, if uncropped.

optical red sequence will be discussed in Section 7. Figure 8 shows the rotated color-luminosity diagram (hereafter referred to as CMD) for the HCGs, with the gap region again highlighted in gray. The color distribution and gap seen in Figure 3 are clearly apparent in Figure 8. Within the HCG sample, a galaxy's luminosity does not appear to depend on its color. Note that even if we relax the luminosity cut $\left(\log \left(L_{4.5}\left[\mathrm{erg} \mathrm{s}^{-1}\right]\right) \geqslant 40.9\right)$, the gap is still quite obvious. Galaxies to the left of the gap have blue MIR colors, indicating that there is no PAH or dust emission and that the MIR SED is consistent with stellar photospheric colors. On the other side of the gap are galaxies with red MIR colors, which means that the SED is dominated by PAHs and warm/ 
Table 4

CDF Slopes in Gap Region

\begin{tabular}{lcc}
\hline \hline \multicolumn{1}{c}{ Sample } & \multicolumn{2}{c}{ Slope } \\
\hline HCG & $\ldots$ & Uncropped \\
LVL+SINGS & 0.732 & 0.104 \\
LVL+SINGS (culled) & 0.896 & 0.743 \\
Interacting & $\ldots$ & 0.883 \\
Coma (center) & 0.600 & $\ldots$ \\
Coma (infall) & 0.261 & 0.518 \\
\hline
\end{tabular}

Note. Slopes of the CDFs of all the samples over the region of the gap in the HCG sample.

hot dust. Galaxies in the gap region would have weak PAH/dust emission compared to starlight. The trend with $\mathrm{H}$ I type is the same as seen in Figure 3: galaxies from H I-rich groups (Type I) generally have "active" (red) MIR colors, while galaxies from H I-poor groups (Type III) generally have "passive" (blue) MIR colors. Galaxies from Type II groups fall on both sides of the gap. Interestingly, galaxies within the gap are from either Type I or Type III groups, but not Type II, but given the small number statistics, this may not be significant.

The CMDs for the comparison samples are shown in Figure 9. Like the HCGs, the luminosities of galaxies in the interacting and both Coma samples also do not appear to be related to color over the range of the sample. However, the LVL+SINGS sample does show a correlation between luminosity and color-the brighter galaxies tend to have MIR-redder colors (with the exception of the "tail"). Thus, LVL+SINGS has primarily faint, MIR-blue (inactive) galaxies and bright, MIR-red (active) galaxies.

As with the color-color plots, the shape of the CMDs reflects the criteria used to define each sample. For example, the center Coma and infall Coma samples have a significant concentration of galaxies with blue MIR colors. These colors indicate SEDs that fall from 3 to $8 \mu \mathrm{m}$, consistent with stellar light arising from the tail of the Raleigh-Jeans distribution, and a general lack of emission from the interstellar medium. The interacting sample, on the other hand, is strongly biased toward actively star-forming systems. Therefore, its CMD lacks a concentration of massive galaxies with blue MIR colors. Instead, it shows a scattering of galaxies with red MIR colors, indicative of PAH features associated with active star formation or SEDs that rise to longer wavelengths due to the presence of warm dust.

The CMD of the HCGs is a composite-it contains both a concentration at blue MIR colors and a scattering of systems with red MIR colors, but very few galaxies with intermediate MIR colors. As suggested by the K-S tests, the HCG CMD distribution is most similar to that of the sample from the Coma Infall region and quite unlike that of the other samples.

\section{PROPERTIES OF GALAXIES ALONG MIR COLOR SPACE}

\subsection{Morphology}

In an attempt to determine the properties of galaxies as a function of MIR color space, as well as the physical origin of the gap, we plotted the distribution of morphologies (obtained from Johnson et al. 2007; Dale et al. 2007, 2009) for the HCG and LVL+SINGS galaxies in color space, as shown in Figure 10. Interestingly, the LVL+SINGS colors appear to be independent of morphology, while the colors of the HCG sample show

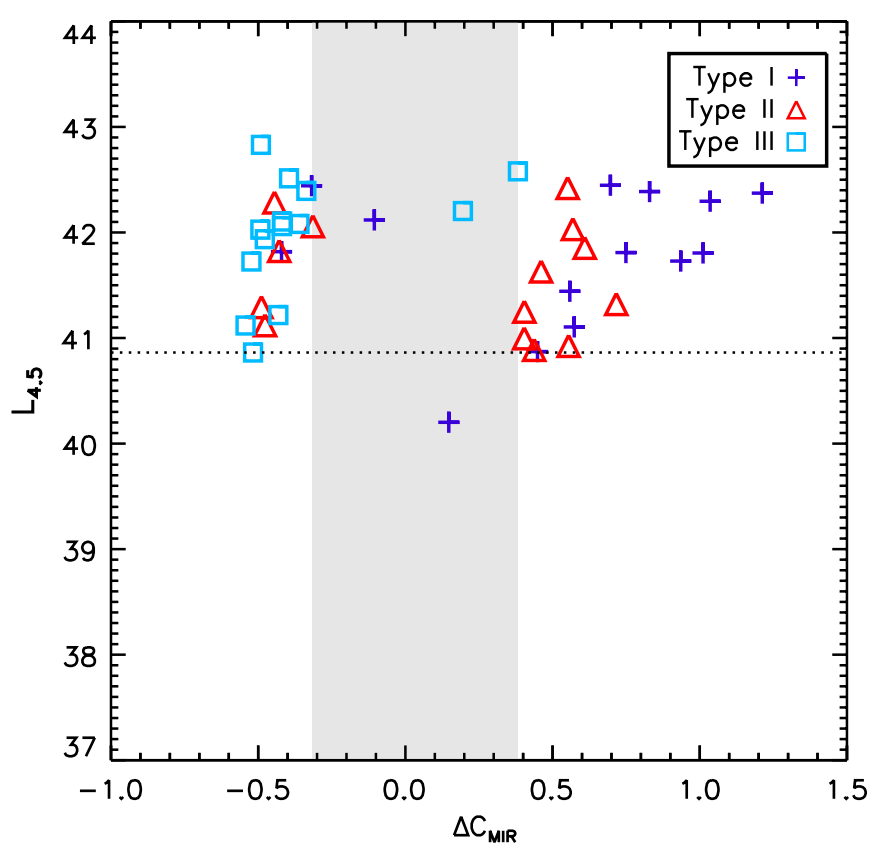

Figure 8. CMD for the HCG galaxies. The dotted line indicates the minimum luminosity required for inclusion. The gap is still apparent, indicated by the gray stripe. Notably, there is no evidence for a color-luminosity correlation.

(A color version of this figure is available in the online journal.)

a morphological segregation. For the HCG sample, the two galaxies that fall in the gap are Sab and Im. The LVL+SINGS sample shows a variety of morphological types in the gap. We considered the possibility that the lack of correlation was due to the diversity of SINGS galaxies, but when we remove the SINGS sample, the same lack of correspondence is seen in the LVL sample. For the culled SINGS sample of Smith et al. (2007), we also do not see a strong correlation between Hubble type and Spitzer IRAC colors.

We find this result both surprising and puzzling, especially given previous studies which have found a trend in MIR color with Hubble type (Pahre et al. 2004) and classical work by Kennicutt et al. (1983) that shows a correlation between $\mathrm{H} \alpha$ equivalent width (EW) and Hubble type. We have investigated the most extreme examples of this lack of morphological segregation-the MIR red E/S0 galaxies and the $\mathrm{Sm} / \mathrm{Sdm}$ galaxies which fall in the gap. We found that the blue early-type galaxies were all starbursts (see Section 6.2), while the red late-type galaxies are low surface brightness galaxies (i.e., NGC 45, 4656, and 5398; Monnier Ragaigane et al. 2003), have low metallicities (i.e., NGC 55; Jackson et al. 2006), or are Seyferts which exhibit large flux variations (i.e., NGC 4395; Minezaki et al. 2006). One possible explanation is that the environment present in CGs causes morphology to more closely track the activity level of a galaxy than in the field. We hypothesize that this could be due to the presence or the absence of neutral gas-if there is gas present, the galaxies will be actively forming stars, which will typically be visible as a disk or irregular galaxy. If most of the gas has been used up, there will be no star formation occurring, and the galaxy will typically be an elliptical or lenticular. In the field, the amount of gas and level of star formation do not appear to be as closely connected, so the galaxy types do not show the segregation seen in the HCG galaxies.

Figure 11 shows Hubble type as a function of rotated MIR color for the HCG galaxies and LVL+SINGS sample. This plot 


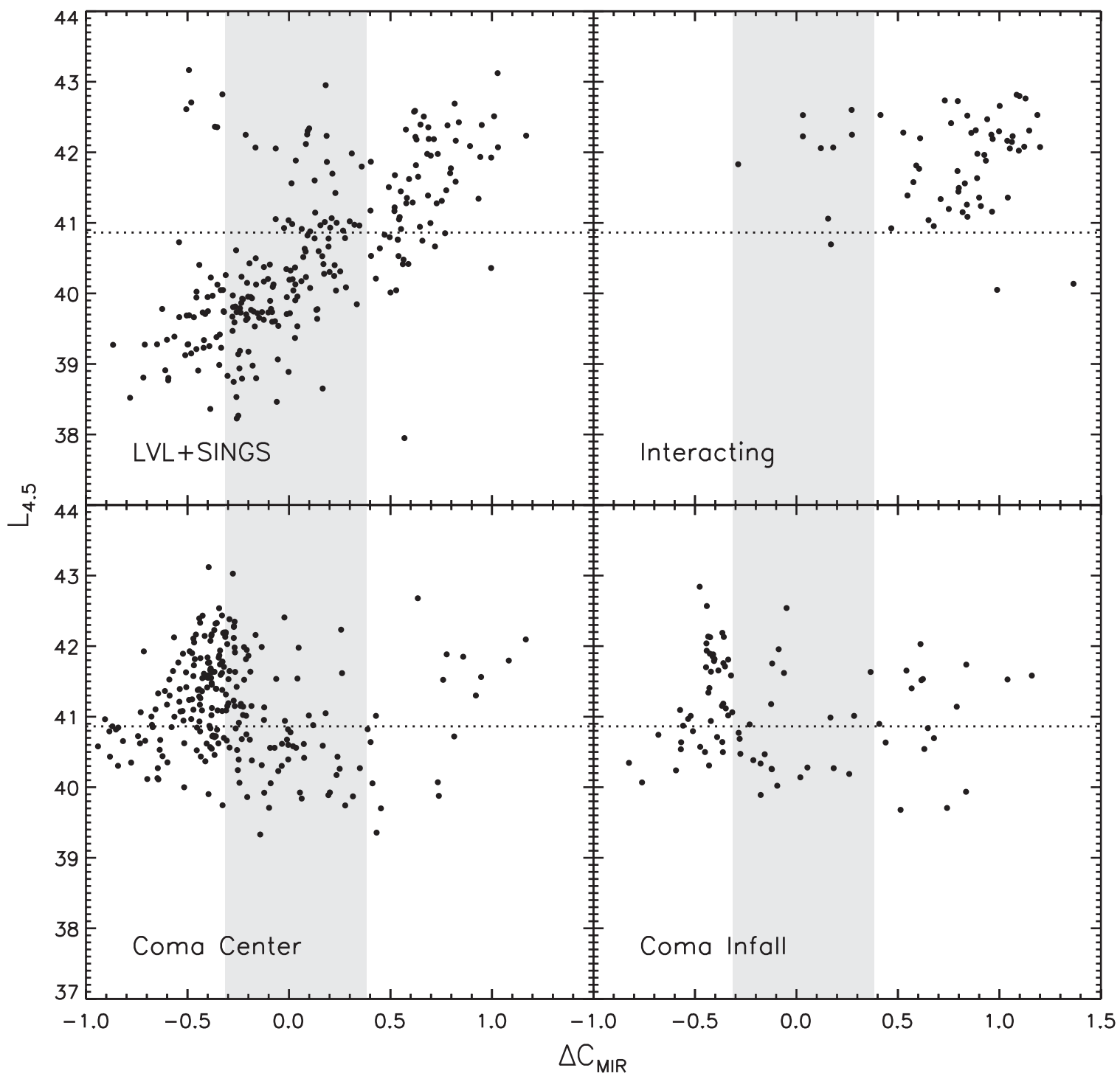

Figure 9. CMD for the comparison samples. As in Figure 8, the dotted line indicates the luminosity cut imposed on the samples, and the HCG gap is highlighted by the gray stripe. The criteria used to define each sample are apparent in the shape of the CMDs. LVL+SINGS is the only sample which shows color dependence on luminosity, discussed further in the text.
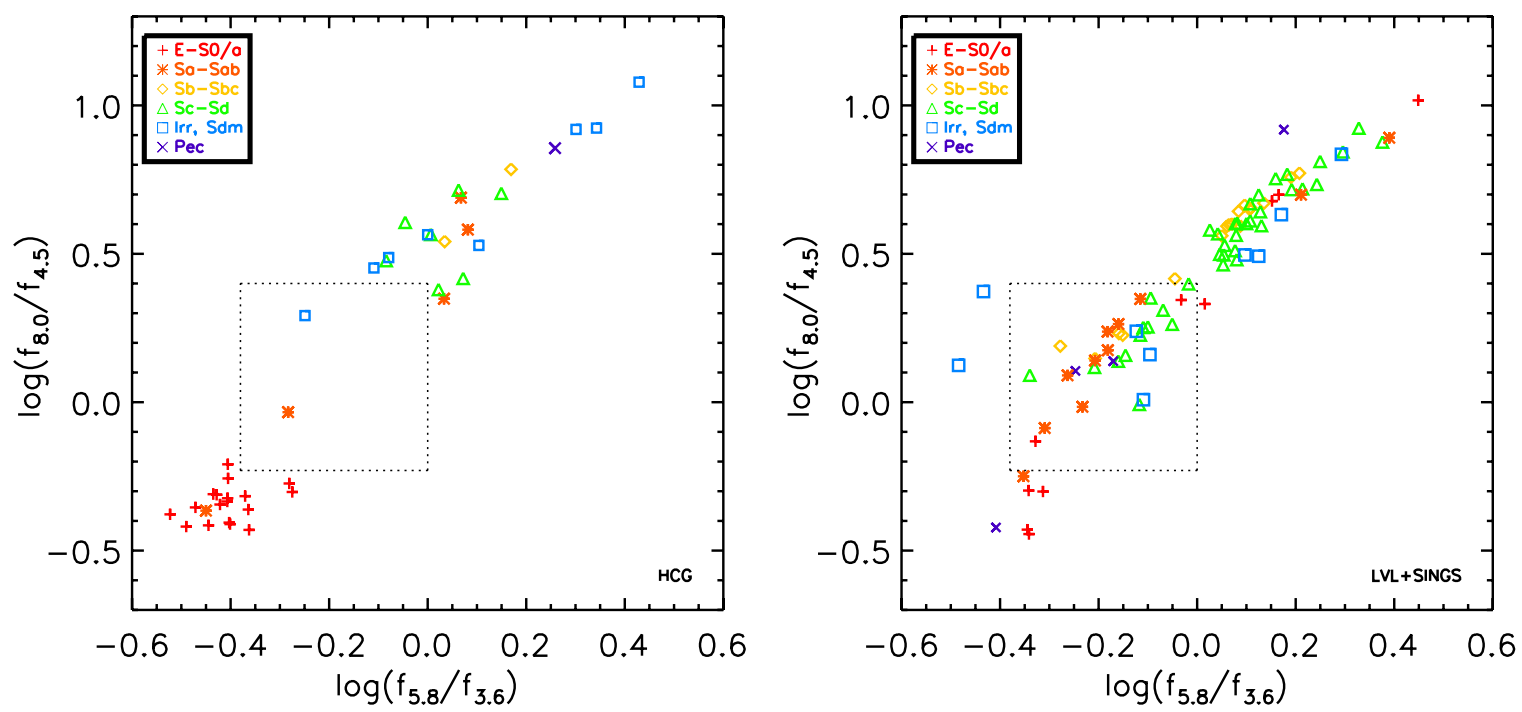

Figure 10. Distribution of galaxy morphology in MIR color space for left: HCGs and right: LVL+SINGS. Note that the distribution of morphologies for the HCGs is as expected, with E/S0 galaxies occupying the lower left and spiral galaxies occupying the upper right. This is not strictly the case for LVL+SINGS. 

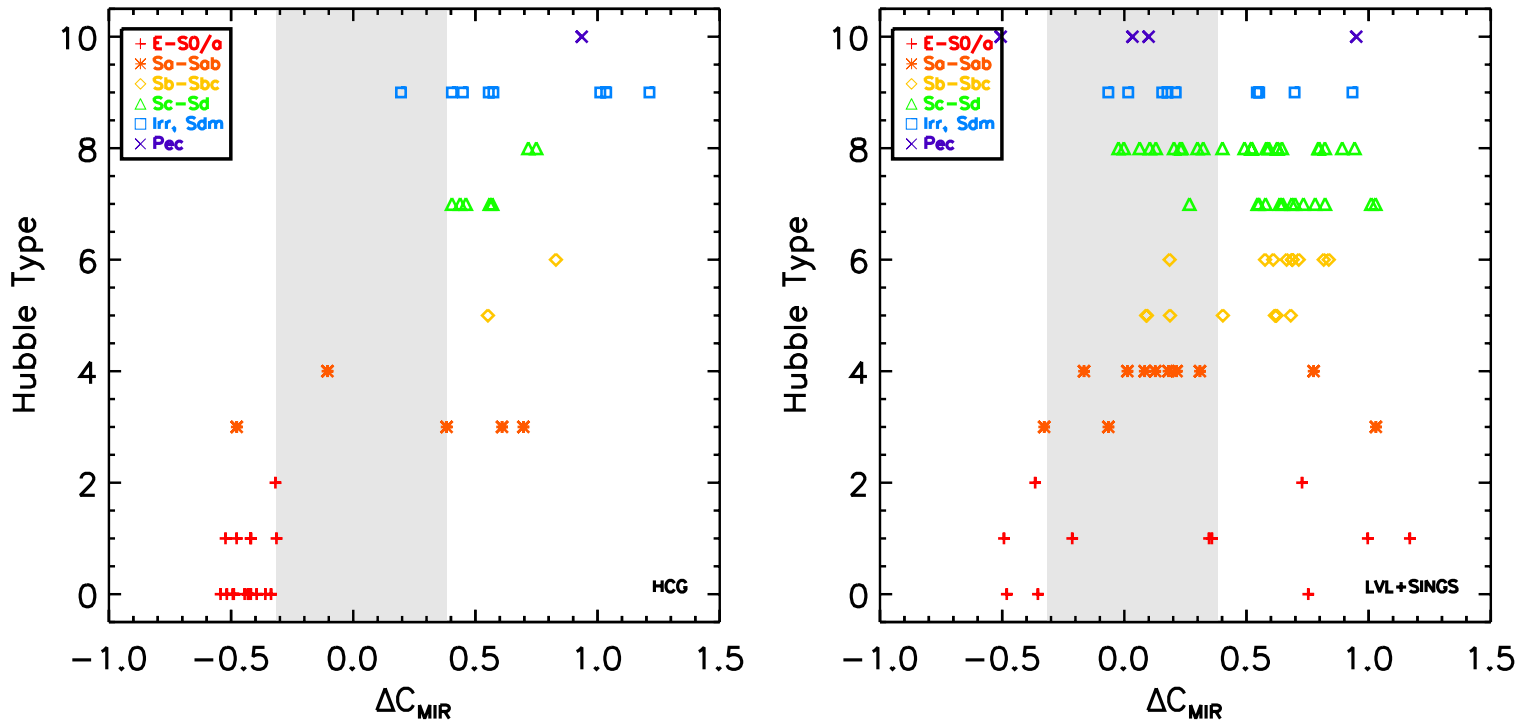

Figure 11. Distribution of galaxy morphology in rotated MIR color space for HCGs (left) and LVL+SINGS (right). Note the dearth of morphologies over $2<T<6$ in the HCG sample.

(A color version of this figure is available in the online journal.)

clearly reveals a dearth of galaxies over $2<T<6$ (S0/a-Sbc) for the HCGs, seen previously in Figure 2. Of the few galaxies with these Hubble types, they do not preferentially fall in the gap. The LVL+SINGS galaxies do not show a dearth of galaxies between these Hubble types. In addition, almost every Hubble type appears in the gap. This begs the question of whether the existence of the gap is nature or nurture. The selection criteria for the HCGs may have for some reason selected against these morphological types. Alternatively, there might something about the HCG environment that suppresses these Hubble types or alters their SFR so that they do not fall in the gap.

\section{2. $E W(H \alpha)$ and Specific Star Formation Rates}

The emission (or absorption) of Balmer lines tracks the recent star formation history of a galaxy through ionized gas and is complimentary to the MIR data which trace warm dust emission. In order to gain more insight into the properties of star formation in the gap region, here we looked at the $\mathrm{EW}(\mathrm{H} \alpha)$ as a function of $\Delta C_{\text {MIR }}$. For four groups in our HCG sample (HCGs 7, 42, 62, 90), we have obtained Hydra CTIO spectra with $2^{\prime \prime}$ fibers located on the nuclei. For many of the galaxies in the SINGS sample, optical spectra were obtained with $2.5 \times 2$ ". 5 slits located on the nuclei (Moustakas \& Kennicutt 2006). These data are publicly available at http://irsa.ipac.caltech.edu/data/SPITZER/SINGS/. In addition to these two data sets, many of the galaxies in both the HCG galaxies and in the LVL+SINGS sample have spectra available through SDSS.

For all of these galaxies, we determined the EW of the $\mathrm{H} \alpha$ line using Gaussians in the SPLOT task in IRAF. Figure 12 shows the morphologies and locations in color space of the galaxies for which we were able to obtain spectra. A plot of EW $(\mathrm{H} \alpha)$ versus $\Delta C_{\mathrm{MIR}}$ is shown in Figure 13. In both the HCG sample and the LVL+SINGS sample, all galaxies blueward of the gap have $\mathrm{EW}(\mathrm{H} \alpha) \lesssim 0$, which is fully consistent with MIR colors indicating predominantly stellar emission. Galaxies redward of the gap exhibit a large range of $\mathrm{EW}(\mathrm{H} \alpha)$, but there is not necessarily a one-to-one correspondence with MIR emission. This could be explained by several scenarios: (1) dust absorption of $\mathrm{H} \alpha$ and general effects of relative geometry;
(2) variable amounts of PAH emission; (3) different timescales traced by $\mathrm{H} \alpha$ and MIR colors; (4) a coverage difference-the MIR colors are integrated over the entire galaxy, while the $\mathrm{H} \alpha$ was measured only in the nuclear region. For a given $\Delta C_{\mathrm{MIR}}$, the galaxies in the LVL+SINGS sample cover a larger range in $\mathrm{EW}(\mathrm{H} \alpha)$ than the HCG galaxies. This is likely because the galaxies in the LVL+SINGS sample have varying amounts of dust-in fact, the SINGS sample was chosen to be as diverse as possible (Kennicutt et al. 2003). All galaxies in the HCG sample have EW $(\mathrm{H} \alpha)$ consistent with their Hubble type (Nakamura et al. 2004). For LVL+SINGS, there are several galaxies which have EW $(\mathrm{H} \alpha)$ inconsistent with their Hubble type-there are peculiar galaxies with $\mathrm{EW}(\mathrm{H} \alpha) \sim 0$ and $\mathrm{E} / \mathrm{S} 0$ galaxies with large EW $(\mathrm{H} \alpha)$. In both samples however, the EW $(\mathrm{H} \alpha)$ seems to exhibit an upper envelope, that changes as a function of $\Delta C_{\mathrm{MIR}}$.

As galaxies in the gap have similar $\mathrm{EW}(\mathrm{H} \alpha)$ in both the HCG and LVL+SINGS sample, the properties of the gap galaxies are likely similar. Hence, the HCG sample must be deficient in a certain type of galaxy, rather than having galaxies with fundamentally different properties. Since the gap is between galaxies with colors consistent with stellar photospheric emission and galaxies with relatively high amounts of star formation, we suggest that the gap is likely caused by a deficit of galaxies with moderate SSFRs. In order to investigate this, we took the SSFRs from Tzanavaris et al. (2010) and plotted them against MIR color, as shown in Figure 14. As is clearly visible from the figure, the MIR gap corresponds perfectly with the gap in SSFR. Thus, the HCG environment appears to be inhospitable to galaxies with moderate SSFRs $\left(4.3 \times 10^{-12}<\right.$ SSFR $\left.<1.6 \times 10^{-11} \mathrm{yr}^{-1}\right)$.

\section{DISCUSSION}

\subsection{The Relationship between Environment and the Presence of Gap}

Our main result is that the HCG sample is statistically different from the comparison samples in MIR color space, $\mathrm{CDFs}$, and rotated CMDs, due to the presence of a gap in MIR color space. Of the other samples considered here, the HCG 

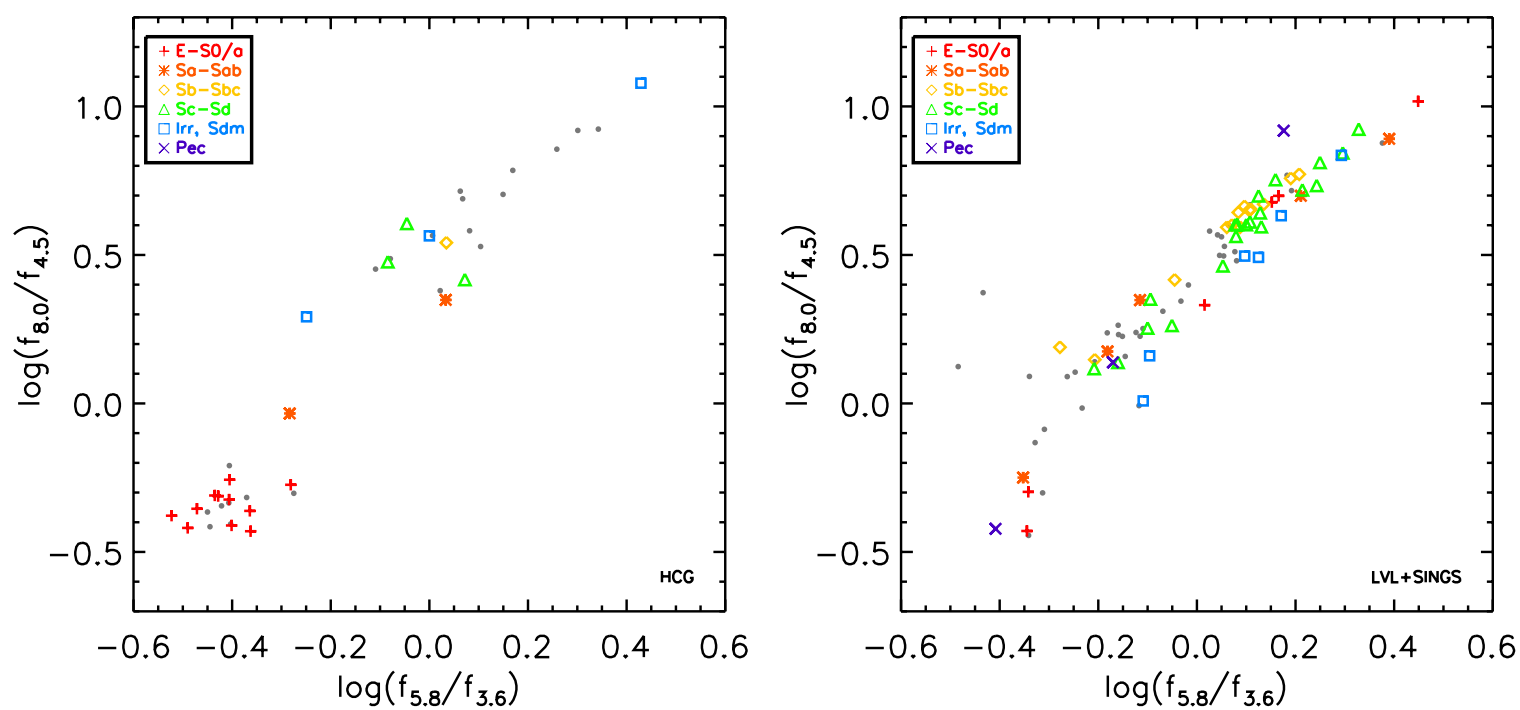

Figure 12. IRAC color space distribution for HCGs (left) and LVL+SINGS (right). Galaxies for which spectra were obtained are identified by their morphological type. Galaxies for which spectra were unavailable are shown as gray dots. These plots show that spectra sample the full range of color space.
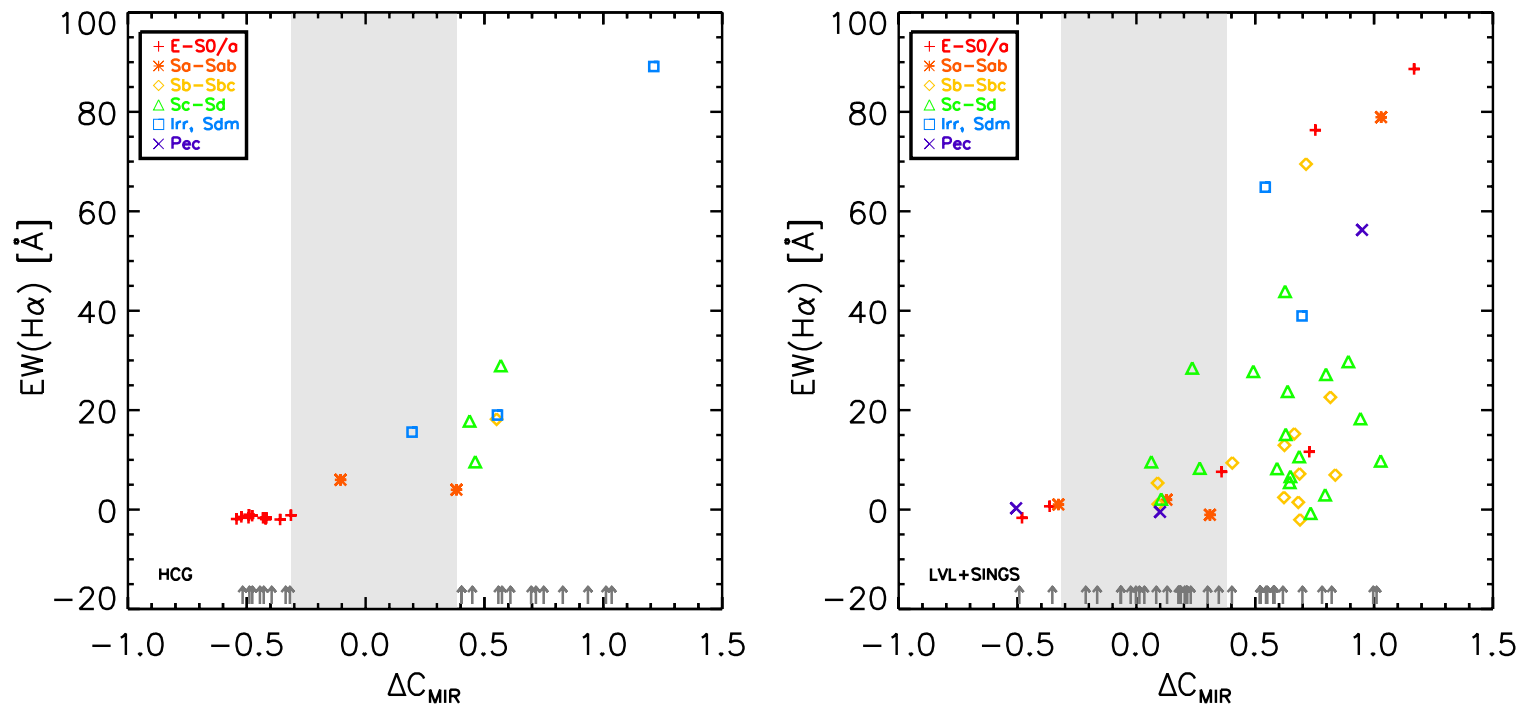

Figure 13. $\mathrm{EW}(\mathrm{H} \alpha)$ vs. $\Delta C_{\mathrm{MIR}}$ for HCGs (left) and LVL+SINGS (right). Galaxies for which spectra were unavailable are plotted as dark gray dots at the bottom. The gap is identified by the gray stripe. In both samples, there is a clear difference between galaxies blueward of the gap and those redward of the gap.

sample is most like the infall Coma sample, and unlike the interacting sample, the center Coma sample, or the LVL+SINGS sample. While the HCG sample is most like the infall Coma sample, the lack of galaxies with transitional MIR colors is more pronounced in the HCG sample.

The fact that the HCG sample is most like the infall Coma sample is unsurprising, as other similarities between the CG environment and the outskirts of clusters have been seen. Lewis et al. (2002) found suppression of SFRs in clusters out to three virial radii and determined that star formation suppression depends more on local galaxy density than on overall cluster properties. In addition, studies by Cortese et al. (2006) of a CG falling into a cluster suggest that the galaxies in the group have undergone pre-processing due to the local CG environment.

\subsection{Possible Mechanisms for MIR Gap}

We have established the statistical significance of the gap in Section 4 , however, the origin and nature of the gap remain an important issue; here, we consider three possibilities. The first is that it arises from a fluctuation due to small number statistics; however, the K-S tests presented in Section 4 conclusively demonstrate that the HCG sample is inconsistent with being drawn from a uniform distribution, even taking into account sample size. The second possibility is a subtle selection effect by either us or Hickson, a possibility that can be investigated by including more HCGs in the analysis as well as expanding the sample to include Redshift Survey Compact Groups (Barton et al. 1996), though it seems unlikely that a selection effect could cause the observed gap. In other words, this would require a bias against galaxies with moderate SSFR, but not low or high SSFR. The third possibility is that the paucity of morphological types could correspond to the MIR gap. The morphology histogram (Figure 2 ) shows a dearth of galaxies between $2 \geqslant T \geqslant 6$. For the HCG sample it is impossible to determine what is not in the gap, but we can look at gap galaxies in LVL+SINGS for insight. Since LVL+SINGS shows galaxies of all types in the gap, it is not consistent with morphological type being a predominant 


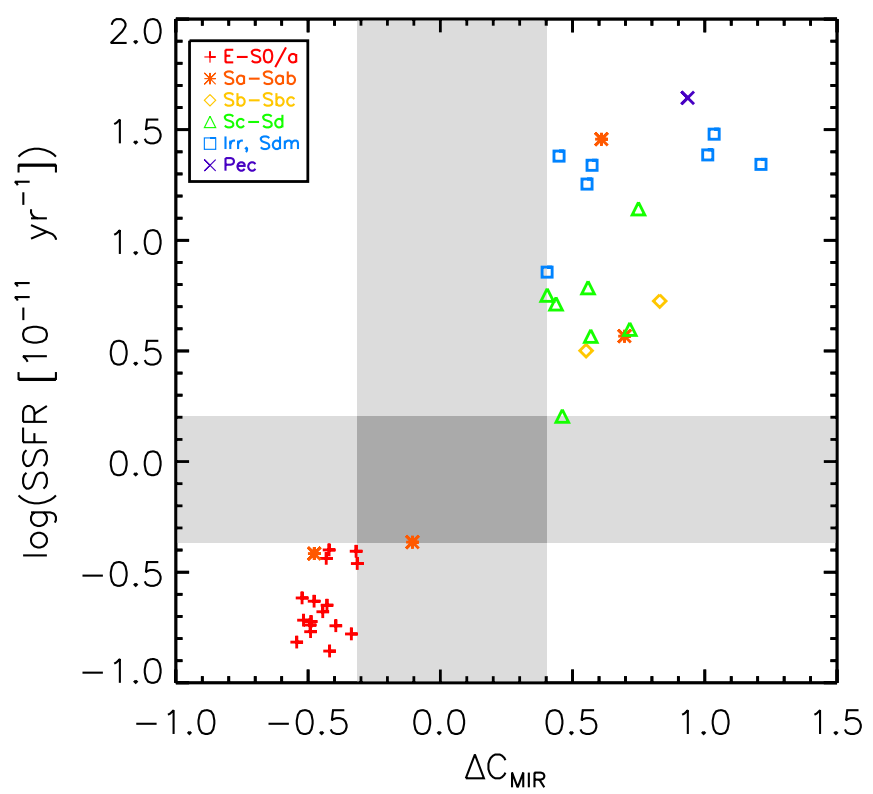

Figure 14. SSFR (from Tzanavaris et al. 2010) vs. $\Delta C_{\mathrm{MIR}}$ for 11 of the 12 HCGs in our sample (HCG 90 was excluded due to insufficient data for determining SSFRs). The vertical gray stripe indicates the gap seen in MIR color space, the horizontal gray stripe indicates the gap in SSFR.

factor in creating the gap. However, it is possible that HCGs select against galaxies that would fall in the gap. This leads us to the possibility that the gap is due to a deficit of galaxies exhibiting moderate SSFRs due to environmental effects of the CG environment.

We conclude that the distribution in color space, and in particular the occurrence of a gap in the MIR color distribution most likely reflects different levels of specific star formation, with galaxies ranging from active (red MIR colors) to passive (blue MIR colors). This is supported by the gap seen in the SSFRs for these HCG galaxies, independently determined using UV, $24 \mu \mathrm{m}$, and $K$-band data (Tzanavaris et al. 2010). The distribution in color space suggests that galaxies in the HCG environment spend little time in the evolutionary state that yields MIR colors in the gap region. The fact that the gap is seen in both the HCG and Coma infall samples, but is more pronounced in the HCG sample indicates that this effect is similar to, but more dramatic than, the environment in the outskirts of clusters. The tidal fields present in HCGs can easily funnel gas to the inner parts of galaxies, inducing high levels of star formation. Once this gas is used up or heated to high temperatures, the galaxies can no longer form stars, and quickly become quiescent. Thus galaxies in HCGs either experience a profusion of star formation or do not have the gas necessary to form stars, and do not exhibit moderate levels of star formation.

\subsection{Relationship to Optical CMDs}

Insight into the shape of MIR CMDs may be gained by analogy to optical CMDs. In the latter, several prominent features have been identified - an optical "red sequence" of galaxies with a relatively narrow distribution of red optical colors, extending to the brightest magnitudes; an optical "blue cloud" of generally fainter galaxies with bluer optical colors (e.g., Strateva et al. 2001; Hogg et al. 2004); and a deficit of galaxies with intermediate optical colors (the so-called green valley; Hogg et al. 2004). Optical red sequence galaxies are dominated by E/S0 galaxies, while optical blue cloud galaxies are dominated by disk and irregular systems. This optical CMD shape is apparent in both dense and sparse environments, although dense environments have a more prominent red sequence, while less dense environments have a more pronounced blue cloud.

The optical CMD shape is often interpreted in terms of evolutionary processes, in which optical blue cloud galaxies might evolve onto the red sequence through some combination of wet and dry merging, star formation, star formation "quenching," and aging (e.g., Faber et al. 2007, and references therein). A key attribute of such models is that galaxies must move rapidly between the optical blue and red phases in order to reproduce the red/blue dichotomy (e.g., Dekel \& Birnboim 2006). The optical "green valley" region therefore includes these evolutionary transitional systems. However, the optical red sequence is contaminated by dusty star-forming galaxies. This is a significant advantage of the MIR over optical.

The interpretation of the MIR CMDs is expected to be similar to that of the optical CMDs, however discussing MIR and optical CMDs simultaneously can be confusing because of the different connotations of "blue" and "red." In optical colors, blue light typically reflects a young population and the optical red sequence picks out both "red and dead" galaxies (i.e., galaxies whose colors are dominated by evolved stars and have little or no active star formation; e.g., van Dokkum 2005; Bell et al. 2004) and galaxies obscured by dust, which may be active. On the other hand, MIR colors reflect the properties of the dust, so blue MIR galaxies select for systems with little dust contribution, which are dominated by stellar photospheric emission (whether from young or old stars), with little or no active star formation. Blue MIR colors therefore select both "optically red and dead" and "optically blue and dying" galaxies. Red MIR galaxies are active, with current star formation heating the dust and exciting the PAHs, so they are most closely related to the optical blue cloud. Additionally, the optical green valley is present in all environments (Hogg et al. 2004), while we have only found evidence for the MIR gap in dense environments that still contain neutral gas.

\subsection{The Role of Gas in CG Evolution}

The presence of neutral gas may be a key factor contributing to the gap in MIR color space. Without fuel for star formation, the MIR colors would be dominated by stellar emission. There are currently two theories on $\mathrm{H}$ I distribution within HCGs: the first is that groups whose $\mathrm{H} \mathrm{I}$ is contained entirely within the member galaxies are less evolved or younger in stellar population than those groups with $\mathrm{H}_{\mathrm{I}}$ that is distributed within and between member galaxies (cf. Williams et al. 1990; Verdes-Montenegro et al. 2001); the second is that the distribution of $\mathrm{H}$ I determines how HCGs evolve, i.e., HCGs whose $\mathrm{H}$ I is contained entirely within the galaxies evolve differently than HCGs whose $\mathrm{H}$ I is distributed throughout the group (Konstantopoulos et al. 2010). Thus, the Hi properties of a group are a crucial component related to a galaxy's MIR color. HCG galaxies from H I-rich groups (Type I) primarily lie redward of the gap, while galaxies from H I-poor groups (Type III) primarily lie blueward. Galaxies from Type II groups lie both redward and blueward of the gap, while avoiding it. Curiously, the few galaxies within the gap are from either Type I or Type III groups. Further investigation into this trend will necessitate interferometric $\mathrm{H}$ I observations of CGs, in order to determine the H I deficiency (and thus type) of individual galaxies within CGs. H I imaging will reveal whether "rogue" galaxies (e.g., MIR-red galaxies from Type III groups) have a different individual deficiency from their group 
as a whole. Interferometric observations will also allow us to determine where the $\mathrm{HI}$ is located-whether it is confined to the member galaxies, or distributed throughout the group. H I distribution could be another clue in $\mathrm{CG}$ evolution.

One estimate of the timescale of the proposed rapid evolution in SSFR is the time it takes a galaxy to use up the available gas and go from starburst to post-starburst. Thus, this could occur on timescales as short as a million years (Gao \& Solomon 1999), but will clearly be highly dependent on environment. A crude upper limit (assuming 100\% efficiency and a constant SFR), using the H I mass of the group divided by the sum of the SFRs in each member galaxy yields gas depletion timescales ranging from 0.4 Gyr to 4 Gyr.

The presence of the gap in the MIR color space distribution of the HCGs combined with the fact that the gap is not present in less dense environments indicates that local environment significantly influences galaxy properties. In order to understand the processes that affect galaxy evolution, we need to understand how gas is processed in the interstellar medium and intragroup medium distinct from the field and cluster environments. CGs are clearly an important part of understanding galaxy evolution and cluster assembly, especially considering the similarities between the distribution in color space of the HCGs and the Coma infall region.

J.C.C. thanks the NSF for funding under award 0908984. K.E.J. gratefully acknowledges support for this paper provided by NSF through CAREER award 0548103 and the David and Lucile Packard Foundation through a Packard Fellowship. S.C.G. thanks the National Science and Engineering Research Council of Canada for support. For helpful discussions on statistical tests, L.M.W. thanks statistics professor Tao Huang. We also thank the anonymous referee for their constructive comments. This research has made use of the NASA/IPAC Extragalactic Database (NED) which is operated by the Jet Propulsion Laboratory, California Institute of Technology, under contract with the National Aeronautics and Space Administration.

\section{REFERENCES}

Barton, E., Geller, M., Ramella, M., Marzke, R., \& da Costa, L. 1996, AJ, 112 , 871
Bell, E., et al. 2004, ApJ, 608, 752

Cortese, L., Gavazzi, G., Boselli, A., Franzetti, P., Kennicutt, R. C., O’Neil, K., \& Sakai, S. 2006, A\&A, 453, 847

Dale, D. A., et al. 2007, ApJ, 655, 863

Dale, D. A., et al. 2009, ApJ, 703, 517

de la Rosa, I., de Carvalho, R., Vazdekis, A., \& Barbuy, B. 2007, AJ, 133, 330

Dekel, A., \& Birnboim, Y. 2006, MNRAS, 368, 2

Faber, S. M., et al. 2007, ApJ, 665, 265

Gallagher, S., Johnson, K., Hornschemeier, A., Charlton, J., \& Hibbard, J. 2008, ApJ, 673, 730

Gao, Y., \& Solomon, P. 1999, ApJ, 512, 99

Haynes, M., \& Giovanelli, R. 1984, AJ, 89, 758

Hickson, P. 1982, ApJ, 255, 382

Hickson, P., Mendes de Oliveira, C., Huchra, J., \& Palumbo, G. 1992, ApJ, 399 353

Hogg, D., et al. 2004, ApJ, 601, L29

Jackson, D., Cannon, J., Skillman, E., Lee, H., Gehrz, R., Woodward, C., \& Polomski, E. 2006, ApJ, 646, 192

Jenkins, L., Hornschemeier, A., Mobasher, B., Alexander, D., \& Bauer, F. 2007, ApJ, 666, 846

Johnson, K. E., Hibbard, J. E., Gallagher, S. C., Charlton, J. C., Hornshemeier, A. E., Jarrett, T. H., \& Reines, A. E. 2007, AJ, 134, 1522

Kennicutt, R. C., \& Kent, S. 1983, AJ, 88, 1094

Kennicutt, R. C., et al. 2003, PASP, 115, 928

Konstantopoulos, I. S., et al. 2010, ApJ, in press, arXiv:1007.3523

Lee, B., et al. 2004, AJ, 127, 1811

Lewis, I., et al. 2002, MNRAS, 334, 673

Mamon, G. 1994, in Clusters of Galaxies, ed. F. Durret, A. Mazure, \& J. Tran Thanh Van (Paris: Editions Frontieres), 291

Mendes de Oliveira, C., \& Hickson, P. 1994, ApJ, 427, 684

Minezaki, T., et al. 2006, ApJ, 643, 5

Monnier Ragaigane, D., van Driel, W., Schneider, S., Jarrett, T., \& Balkowski, C. 2003, A\&A, 405, 99

Moustakas, J., \& Kennicutt, R. 2006, ApJS, 164, 81

Nakamura, O., Fukugita, M., Brinkmann, J., \& Schneider, D. 2004, AJ, 127, 2511

Pahre, M., Ashby, M., Fazio, G., \& Willner, S. 2004, ApJS, 154, 235

Ribeiro, A., de Carvalho, R., Capelato, H., \& Zepf, S. 1998, ApJ, 497, 72

Smith, B., Struck, C., Hancock, M., Appleton, P., Charmandaris, V., \& Reach, W. 2007, AJ, 133, 791

Strateva, I., et al. 2001, AJ, 122, 1861

Tzanavaris, P., et al. 2010, ApJ, 716, 556

van Dokkum, P. 2005, AJ, 130, 2647

Verdes-Montenegro, L., Yun, M., Williams, B., Huchtmeier, W., del Olmo, A., \& Perea, J. 2001, A\&A, 377, 812

Williams, B., McMahon, P., \& van Gorkom, J. 1990, NASA Conference Publication, Vol. 3098, Paired and Interacting Galaxies, 93

Wilman, D., et al. 2008, ApJ, 680, 1009 